\title{
Research on the Aesthetic Experience of Consumers in Product Design
}

\author{
Bing-qian Wu
}

\author{
Department of Mechanical Engineering, North China Electric Power University, Baoding, China \\ 549645425@qq.com
}

Keywords: Product design, Aesthetic experience, Visual aesthetic, Emotional response

\begin{abstract}
Consumer aesthetic experience in product design can influence his/her product perception and purchasing behavior. The mechanism of aesthetic experience involves three parts, research on neuroscience, information processing model and process of emotional responses. Specific figures and structures, generalized design elements and individual factors may influence consumers' aesthetic experiences in product design. Furthermore, consumers' aesthetic experiences may influence perceived product performance, product evaluation and purchase intention through spillover effect, contagion effect and contrast effect. Future research should solve the contradictions among existing literatures, and continue to explore the theoretical and managerial implications of aesthetic research in the field of consumer behavior.
\end{abstract}

\section{Introduction}

As the core attributes of the product become more homogeneous, companies begin to shift their differentiation efforts from specific product characteristics to some non-tangible features, such as product design aesthetics [1]. In many cases, product design can not only play a functional value with the consumer information exchange, but also can provide an aesthetic appreciation of the value of pleasure. Product design aesthetic and symbolic value is an important factor affecting the choice of consumer products [2]. And product strategy has important practical significance. Product design provides two kinds of interests for consumers, including enjoyment and practicality. Aesthetics is an important aspect of the enjoyment of the product. Consumers can enjoy the aesthetic value of the aesthetic experience from the products with aesthetic appeal. The products with aesthetic appeal tend to have higher product evaluation than the standardized products. When the practical benefits of the product exceeds the expected, it will lead to consumer satisfaction with the product and brand, but can not guarantee brand loyalty; and when the product benefits exceeds the expected, it will make consumers on the product or brand holding more high loyalty. It can be seen that, as a product of the enjoyment interests, aesthetics can enhance consumer loyalty to the brand [3].

However, in the field of aesthetic design of product, there is no systematic scientific theory to guide practice. In the enterprise product strategy, the manager from the aesthetic point of view of product design guidance is only in a stage of experience and intuition. The decision-making has been a lack of scientific basis. The existing theoretical research is still fragmented, the correlation between the research and continuity is relatively low. Therefore, it is necessary to systematically sort out relevant theories and research findings in order to provide a basis for future research in this field, as well as to make research gaps and research deficiencies more clearly presented to consumer behavior researchers, thus promoting the field theoretical development.

\section{The Concept and Mechanism of Aesthetics}

The Concept of Aesthetics. Aesthetics refers to the visual form and sensory experience of objects associated with structure, coordination, rules, and beauty. Aesthetics is through human vision to influence people's perception of product design and behavior. Vision is the human feeling of the five kinds of feelings in the largest amount of information, the most obvious impact on the consumer a feeling. The visual aesthetics of product design can make the product more expressive, inspiring and pleasant[4]. Usually, the aesthetic value and the value of pleasure are simultaneous, and successful product design often provides these two values to the consumer. 
The Mechanism of Aesthetic Experience. The concrete mechanism is shown in Fig 1 which includes three aspects.

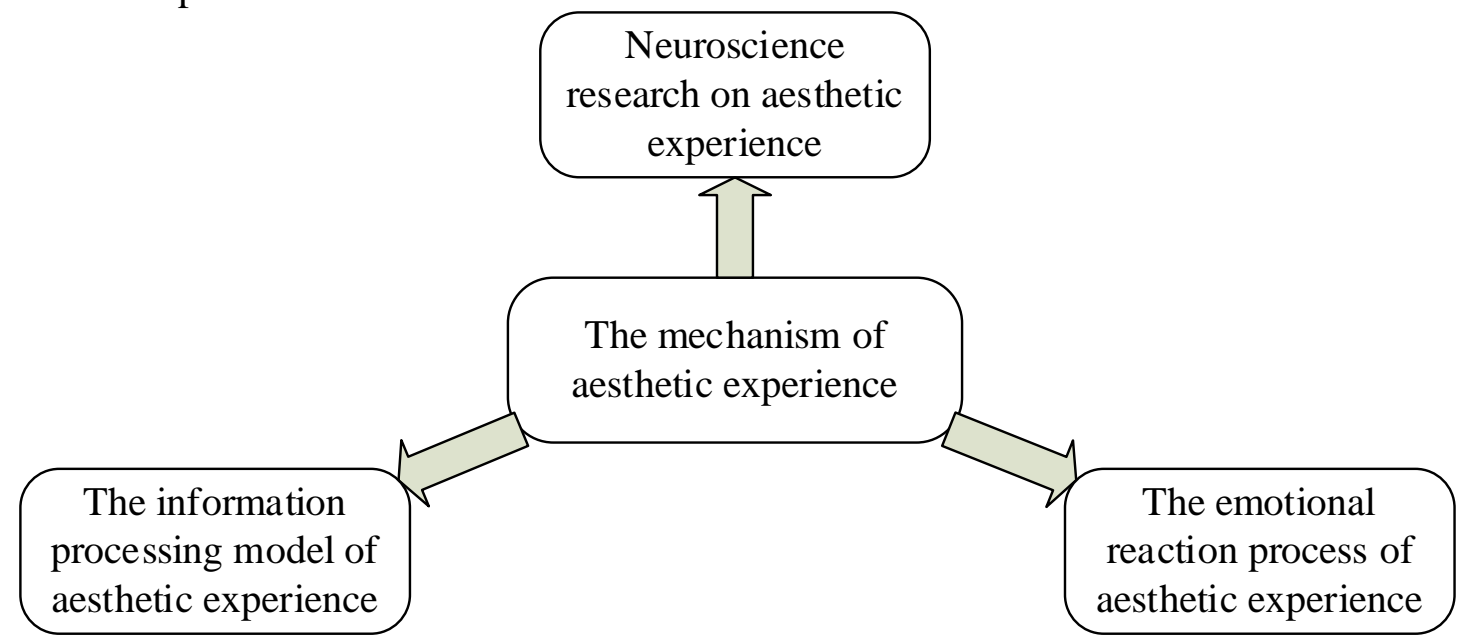

Fig 1. The mechanism of aesthetic experience

Neuroscience Research on Aesthetic Experience. Aesthetic experience has the value of pleasure, which is a self-reward to provide a cognitive process. Neuroscience studies have confirmed that aesthetically pleasing visual stimuli associated with ugly visual stimuli in the brain associated with higher reward values. The understanding of the works of art will result in the activation of the center of the reward in the brain, and the activation of the reward center will affect people's aesthetic preferences, judgments and subsequent decisions. The higher the aesthetic value of the product packaging design, the easier it is to stimulate the reward system in the brain, thereby increasing the product evaluation and preferences.

The Information Processing Model of Aesthetic Experience. Leder et al. developed the information processing model of aesthetic experience[5]. The aesthetic experience in this model includes five stages: perception analysis, implicit memory integration, explicit classification, cognitive control and evaluation. According to their point of view, aesthetic experience is a cognitive process, it is accompanied by rising emotional state to trigger aesthetic feelings. Art is the classic field of aesthetic research, and the exposure to art provides a challenging scene for the senses. The perceptual person succeeds in classifying, understanding and conquering the artwork. The whole process is the aesthetic experience.

The Emotional Reaction Process of Aesthetic Experience. Consumer response to product design can be divided into cognitive and emotional categories. Emotional response in the price can be divided into positive and negative two categories[6]. The aesthetic response to product design is a positive emotional response, which stems from a strong positive emotion and pleasure. These positive feelings arise in the form of consciousness or unconsciousness in interaction with objects. Aesthetic response stems from the design and product sense of the property, rather than functional attributes. For example, the purchaser of a high-end porcelain obtains an aesthetic experience from the appearance of porcelain rather than durability.

\section{The Antecedents and Consequences of Aesthetic Experience}

This section is consist of three parts shown in Fig 2.

The Important Design Factors Affecting Aesthetic Experience. Many product design factors will significantly affect the consumer's aesthetic experience [7]. These design factors include both the specific graphics and structural elements of the combination and match, but also contains the general product design factors, shown in Fig 3.

Graphics and Structure. Graphic elements (eg, colors and trademarks) and structural elements (eg, shape, size, and material) are important design factors that affect the consumer's aesthetic experience[8]. Studies have examined the effects of color on aesthetic preferences, the combination of specific shapes and colors, the color combination preferences, and the symmetry preferences. If a 
particular symbol is consistent with the cultural values and moral values of a group, the object containing the sign is attractive to the group.

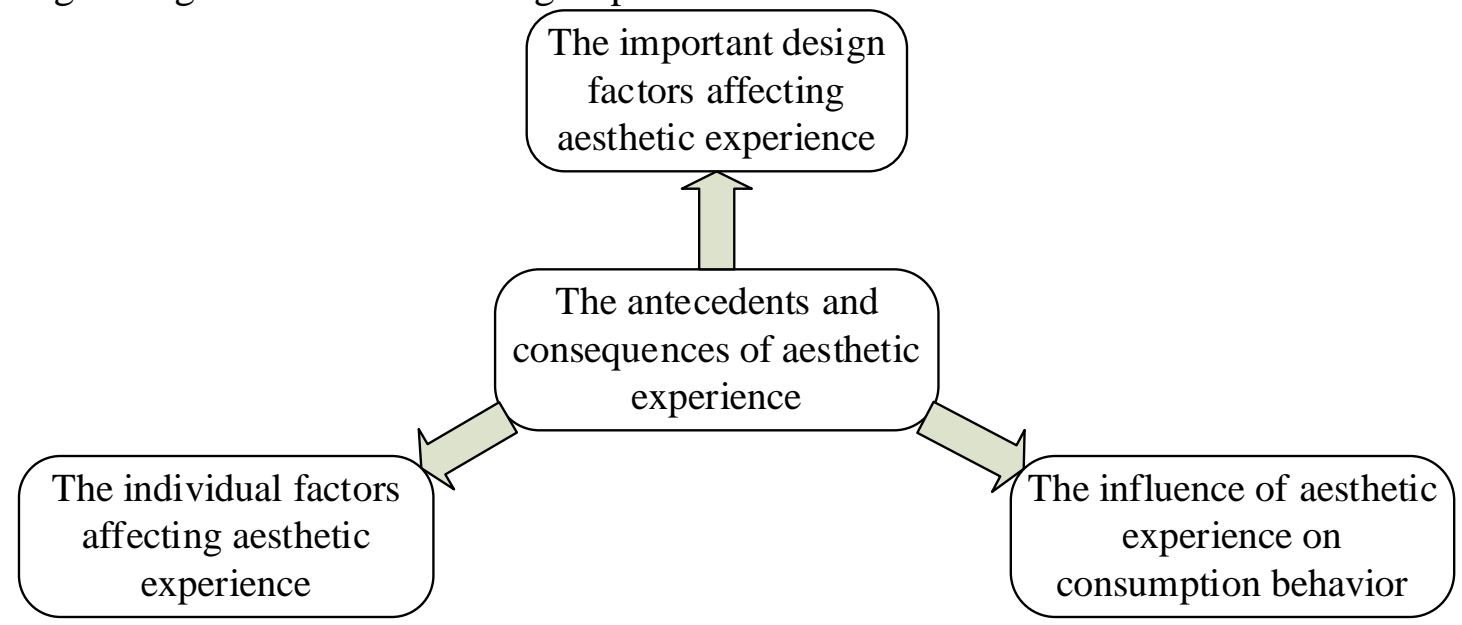

Fig 2. The antecedents and consequences of aesthetic experience

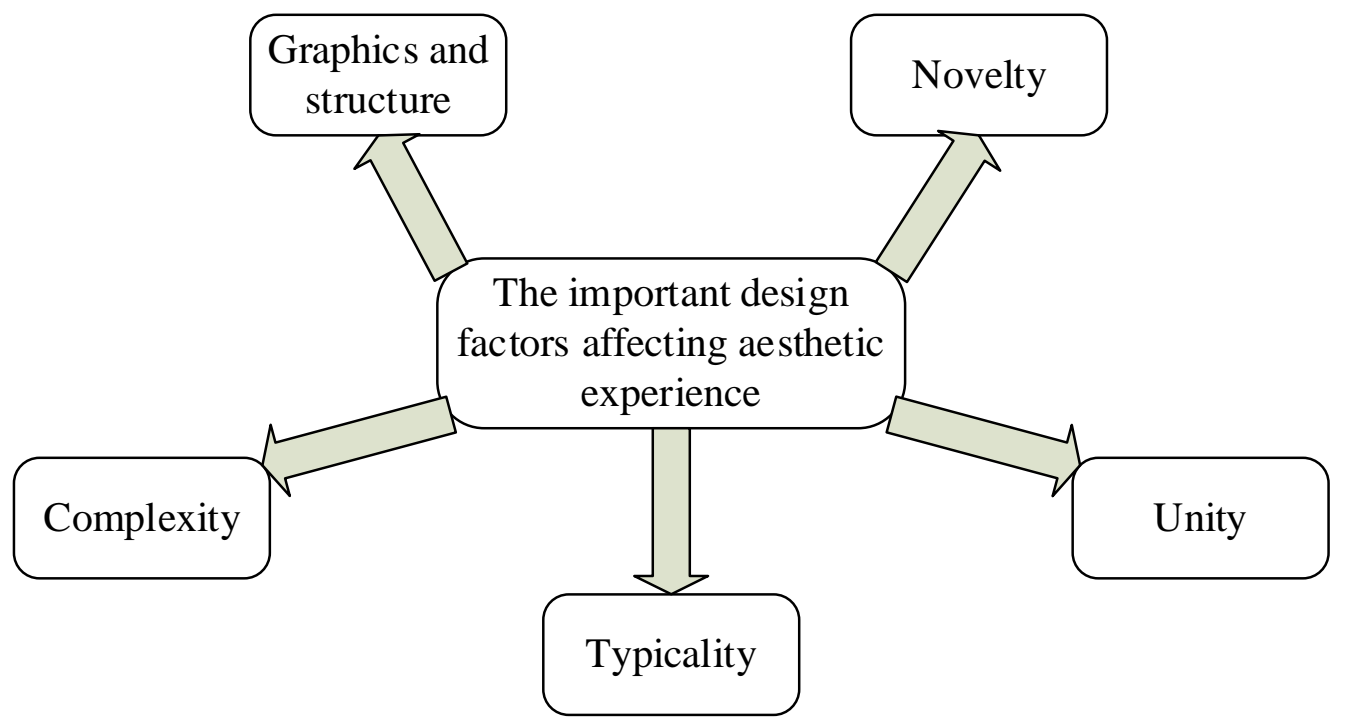

Fig 3. The important design factors affecting aesthetic experience

Novelty. Novelty means that, based on the comparison of the current form to the past experience form, the graphic units and their organization are perceived novel. In product design, novelty has an important impact on the evaluation of product appeal[9]. Consumers will prefer different levels of the product, the consumer's preference for novelty is inverted U-shaped. In the form of aesthetics, each nation or country has a different preference for novelty, because culture influences people's preference for novelty.

Complexity. Complexity refers to the degree of stimulation from the number of graphic units and the quality of the entity, the degree of difference between the graphics units, and the degree of organization of these graphic units [10]. The classical aesthetics theory points out the inverted U-type relationship between pleasure and complexity, and achieves the highest level of pleasure at a moderate level of complexity. Low complexity of the object has a low level of stimulation, compared with the moderate level of complexity caused by a little pleasure; and high complexity will lead to confusion, the same cause less pleasure.

Typicality. The typicality is defined as the degree to which an object can represent a category. From this perspective, the typical is seen as a good example of a measure, a typical object is best used to represent a class of objects. The typical design of the product will significantly affect the consumer's aesthetic experience. In the past there was a difference in the impact of the typicality on aesthetic evaluation[11]. Part of the study argues that people prefer typical objects to atypical objects because people tend to use objects that are consistent with their current knowledge. Other scholars believe that atypical product design is more attractive than typical product design. 
Unity. Unity means that there is consistency between the design elements, these design elements appear to be a whole or there is a visual contact rather than accidentally being aggregated together. Recent studies have found that unity as an important design element will significantly affect the user's perception of computer interface aesthetics[12]. The combination of aesthetic elements will significantly affect people's perception, when the product design of the elements of a higher unity when consumers can get a higher aesthetic experience.

The Individual Factors Affecting Aesthetic Experience. From the consumer point of view, the perception and response of different consumers facing the same product design are different, depending on the characteristics of consumers themselves. Many consumer personality traits affect aesthetic experiences such as personality, lifestyle, psychometric variables, and demographic variables (income, educational level, occupation, gender, age, etc.). For example, women and men have a difference in the judgment of aesthetics. Men prefer more intense colors, heavier materials and rectangles, while women prefer soft colors, soft texture and round[13]. Some of the feelings of aesthetics mainly through the natural feeling, while others aesthetic judgment is mainly affected by the culture. However, how these consumer personality specifically affect consumer behavioral response to aesthetic product design has not been fully explored.

The Influence of Aesthetic Experience on Consumption Behavior. Aesthetic appeal of the product will be a positive brand evaluation. If the two products are similar in function and price, then consumers will prefer the kind of aesthetic appeal to bring pleasure to the people of the product[14]. Visual aesthetics will bias consumers' perceptions. Aesthetic context (such as packaging) will lead to perceived bias because people will notice the prominent appearance of the characteristics. Although aesthetic cues are not diagnostic in terms of quality, the response to aesthetics leads to more diagnostic clues[15]. Some scholars found in the experiment, even if the product evaluation is based on the actual taste, brand labels or product color visual cues or will affect the ability of consumers to distinguish quality.

\section{Conclusion}

It can be seen from the combing of the above discussion that the research in this field mainly involves the internal mechanism of the aesthetic experience, how the enterprise designs the product from the aesthetic point of view, how the aesthetic experience affects the product evaluation and the purchase, the consumer's personality in these processes effect. Product design visual aesthetics is one that has not yet been fully developed

In the field of research, scholars have no uniform view of the object and content of the research problem, the degree of contact between the research is low, the study fragmented system. Future research needs to establish theoretical position for all kinds of research in this field, so that the field of theoretical development has a clearer context. In the field of visual aesthetics of product design, there are many contradictions in the research results. For example, some studies suggest that the higher the aesthetic appeal of the product's appearance, the better the product evaluation through the spillover effect, and the research advocates to improve the product evaluation by reducing the aesthetic appeal of the product packaging and creating a positive emotional response. Some scholars advocate by enhancing the novelty of the product to enhance aesthetic evaluation. Novelty and familiarity are relative, and some scholars believe that the increase in familiarity will increase the preferences of aesthetic objects. In general, there are still many contradictions between the various studies in the field that need to be addressed.

In short, product design aesthetics will give consumers an aesthetic experience and thus affect consumer awareness, attitudes and behavior, product design aesthetics in the enterprise product strategy plays a decisive role, is to win the consumer and the market key factors. The urgent need in practice requires the academic community to give enough attention in this field, and the scholars need to further explore the mysteries of product design aesthetics and consumer aesthetic experience. 


\section{References}

[1] Alpert, J. I., Alpert, M. I. Music influences on mood and purchase intentions[J]. Psychology \& Marketing, 1990, 7(2): 109-133.

[2] Silvia, P. J. Emotional responses to art: From collation and arousal to cognition and emotion[J]. Review of General Psychology, 2005, 9: 342-357.

[3] Blijlevens, J., Carbon, C. C., Mugge, R., Schoormans, J. P. L. Aesthetic appraisal of product designs: Independent effects of typicality and arousal[J]. British Journal of Psychology, 2011, 103(1): 44-57.

[4] Venkatesh, A., Joy, A., Sherry, J. F., Jr, Deschenes, J. The aesthetics of luxury fashion, body and identify formation[J]. Journal of Consumer Psychology, 2010, 20: 459-470.

[5] Leder, H., Belke, B., Oeberst, A., Augustin, D. A model of aesthetic appreciation and aesthetic judgments[J]. British Journal of Psychology, 2004, 95(4): 489-508.

[6] Brunel, F. F., Swain, S. D. A moderated perceptual model of product aesthetic evaluations[J]. European Advances in Consumer Research, 2008, 8: 444-445.

[7] Chattaraman V., Rudd N. A., Lennon S. J. The malleable bicultural consumer: Effects of cultural contexts on aesthetic judgments[J]. Journal of Consumer Behavior, 2010, 9: 18-31.

[8] Hagtvedt, H., Patrick, V. M. Art infusion: The influence of visual art on the perception and evaluation of consumer products[J]. Journal of Marketing Research, 2008, 45: 379-389.

[9] Creusen, M. E. H., Veryer, R. W., Schoormans, J. P. L. Product value importance and consumer preference for visual complexity and symmetry[J]. European Journal of Marketing, 2010, 44: 1437-1452.

[10] de Tommaso, M., Pecoraro, C., Sardaro, M., Serpino, C., Lancioni, G., Livrea, P. Influence of aesthetic perception on visual event-related potentials[J]. Consciousness and Cognition, 2008, 17: 933-945.

[11] Chitturi, R., Raghunathan, R., Mahajan, V. Delight by design: The role of hedonic versus utilitarian benefits[J]. Journal of Marketing, 2008, 72: 48-63.

[12] Armstrong, T., Detweiler-Bedell, B. Beauty as an emotion: The exhilarating prospect of mastering a challenging world[J]. Review of General Psychology, 2008, 12: 305-329.

[13] Winkielman, P., Halberstadt, J., Fazendeiro, T., Catty, S. Prototypes are attractive because they are easy on the mind[J]. Psychological Science, 2006, 17 (9): 799-806.

[14] Pan, S., Tsai, H., Lee, J. Framing New Zealand: Understanding tourism TV commercials[J]. Tourism Management, 2011, 32: 596-603.

[15] Landwehr, J. R., Wentzel, D., \& Herrmann, A. The influence of prototypicality and level of exposure on consumers' responses to product designs: Field evidence from German car buyers[J]. Advances in Consumer Research, 2010, 37: 682-683. 